\title{
Intramedullary nailing of femural shaft fracture
}

\author{
Mehmet Ata Gökalp*, Seyyid Şerif Ünsal, Abdurrahim Gözen, Necip Güven, Savaş Güner \\ Department of Trauma and Orthopedic Surgery, Medical School of Yuzuncu Yil University, Van, Turkey
}

\begin{abstract}
In this study, we examined adult patients with femur shaft fracture who treated with open-closed reduction and reamerized intramedullary nailing. Thus we aimed to evaluate clinical and radiological results of the adult patients and compare them with the studies in the literature.

42 extremities of 40 patients ( 35 male- 5 female) who admitted to Yüzüncü Yil University Faculty of Medicine, Department of Orthopeady and Traumatology with femur shaft fracture and were treated with locked intramedullary nail between 2009 and 2012 are included in this study.

We aimed to evaluate early results of locked intramedullary nailing. In light of these evaluations we saw that our results were compatible with the literature. We believe that locked intramedullary nailing is one of the most successful methods in the treatment of femur shaft fractures.
\end{abstract}

Key Words: Fractures of femur, intramedullary nail, clinical result

\section{Introduction}

Femur shaft fractures are the general name of femur fractures which starts $5 \mathrm{~cm}$ beneath the trochanter minor and reaches up to the proximal of the adductor tubercle (1). These fractures usually emerge after high-energy traumas and are frequently seen in young adults. In traumatology, these kinds of fractures are encountered very often and they cause significant mortality and morbidity (2). These fractures may be life threatening in cases like open wound, fat emboli, acute respiratory distress syndrome and multiple organ failure (3-4).

Main goal in the treatment of femur shaft fracture is to provide anatomical integrity and regaining early functions. Due to the multitude of complications (common osteoporosis, sudeck's atrophy, stone in the urinary system, joint stiffness etc.) encountered in conservative treatment of femur shaft fractures in adults, surgical treatment is preferred $(2,5-7)$.

Similar to all bone shaft fractures, femur shaft fractures can be treated with four main methods: conservative treatment (traction and plastering), external fixation method, plate fixation and intramedullary nailing. Even if conservative treatment is a rarely used method in adult patients, traction can be an option in patients who have no chance of surgical treatment, are old, mental reterdation, and have additional problems. Due to the success and common recognition of the intramedullary nailing, plate fixation that enables rigid fixation and early mobilization is only and rarely seen in the treatment of femur shaft fracture in adults at the present time. External fixation operations are used at the first stage of the treatment of the patients who have unstable open fractures Also they are used as the start-up phase of the treatment in open fractures in situations where severe soft tissue damage. Even if the intramedullary nailing started to be used between 1950 and 1960, it gained its popularity in the 1980s with locked nails. Moreover it has been a universal treatment standard due to the high rates of nonunion that reached up to $98 \%$ and low infection risk even in open fractures (1). In comparison to conventional nails, locked nails which blocks shortness and bad alignment in the later processes, are the most important underlying causes of the success of the method (1).

In this study, we examined adult patients with femur shaft fracture who we treated with openclosed reduction and reamerized intramedullary nailing. Thus we aimed to evaluate clinical and radiological results of the adult patients and compare them with the studies in the literature.

\section{Materials and methods}

42 extremities of 40 patients [35 male-5 female] who admitted to Yüzüncü Yıl University Faculty of Medicine, Department of Orthopeady and Traumatology with femur shaft fracture and were 
treated with locked intramedullary nail between 2009 and 2012 are included in this study. The patients with open fracture (except type 1-open fracture) were excluded from the study after wound care and antibiotherapy. Also the patients who underwent intramedullary fixation after being followed up with external fixator were not included in the study. The mean age was 31 [age distribution: 18-57] and average follow-up time was 21.4 months [9-36 months]. The extremity where fracture was developed was right fracture in 24 patients, left fracture in 14 patients and bilateral fracture in 2 patients.

When we classify the fractures by dividing the region from the trochanter minor to the adductor tubercle, We saw that there was 4 patients in proximal femur fractures and 22 patients in shaft fractures and 16 patients in distal fractures. When we classify the fractures according to the Winquist and Hansen classification (8), we found that 4 patients had "type 0" fracture, 12 patients had "type 1" fracture, and 26 patients had "type 4" fracture. According to the open-closed fracture classification of Gustillo-Anderson, 31 were closed and 11 were type- 1 open fracture out of 42 extremity fractures.

The trauma that caused fracture was traffic accident in 23 patients, fall from height in 10 patients, gunshot wounds in 6 patients and work accident in 1 patient. The reason for fracture was high-energy trauma in almost all patients. In addition to the femur fracture, there was humerus fracture in 2 patients, distal end radius fracture in 2 patients, ulnar shaft fracture in 2 patients, fracture of the opposite femur neck in 1 patient, tibial shaft fracture in 1 patient, lumbar vertebra fracture in 2 patients, thoracic vertebra fracture in 1 patient, neurological deficit in 2 patients, vascular injury in 2 patients, contusion in brain in 2 patients, and maxillofacial trauma in 1 patient.

The patients were evaluated by the clinics of general surgery, thoracic surgery, orthopedics, and neurosurgery service with a multidisciplinary approach when they admitted to the emergency department of the hospital. Routine radiographies and also bilateral radiographies of the extremity where there could be fractures were taken.

Until the surgery, skeletal traction was applied to the patients from the tuberositas tibia. They were followed up by strapping loads that did not exceed $10 \%$ of their body weights. After the application of the procedure for treatment open fractures (tetanus prophylaxis, recurrent wound debridement, considering sufficient time for antibiotherapy, internal fixation after temporary external fixation) open fractures (except type-1 fracture) were excluded from our study since they were followed-up with intramedullary or external fixation. While in closed fractures antibiotheraphy was not applied, in all open fractures empiric antibiothreapy which is effective against gr (-), gr $(+)$ and anaerobic microorganism was used. All patients treated with low molecular weight heparin and antithrombotic medication during the time that they were in the hospital and in the postoperative period.

Before the operation, the radiographies of the unproblematic/healthy parts were taken from all patients and the distance between trochanter major and lateral condyle was measured. Thus we formed an opinion about the nail length and the width of medullary canal. During the operation all nail lengths were made available. All of the patients were used radiolucent operation table with scopy-guided imaging. Distal nails were locked by free hand technique, via scopy guided intervention in patients. In segmental fractures, the segments were tried to stick together by cerclage wire. In order not to disrupt the feeding of fragments, periosteum was protected as far as possible. Based on the Winquist and Hansen classification, in type 0-1-2 fractures, dynamic locking and one distal locking screw was used. On the other hand, in type 3-4 cases, static locking and two distal locking screws were used. Incisions were closed by placing hemovac drain.

Drains were removed in the second postoperative day by dressing the wound. The knee, hip, and joint movements and exercises that would strengthen quadriceps were given to the patients in the earliest periods that they could tolerate. In the patients whose general medical condition did not pose a problem, partial weight was given at the level that they could tolerate with crutch. It was recommended to take out stitches at the $15^{\text {th }}$ day. The patients invited for the control once in a month for six months and the future controls scheduled quarterly (every 3 months) for six months and semiannually for the next six months.

\section{Results}

The average follow-up time was 21.4 [9-36] months. The patients were operated averagely after 2.6 days. In the postoperative period, empiric antibiotherapy was applied in closed fractures for two days and in open fractures for 7 days. The average duration for length of hospital stay was determined as 6.9 days [3-10].

East J Med Volume:21, Number:1, January-March/2016 
General anesthesia was applied to 15 patients and spinal anesthesia was applied to 25 patients. In 8 patients, closed reduction, in 32 patients open reduction with mini incision was performed. Then the nail was placed.

We allow early weight bearing to the patients who have dynamic locking. Neurovascular deficit did not develop in any of the patients. In the last control, we controlled the existence of pain, swelling and shortening. We examined knee joint range of motion and, the existence and angle of rotation. Then we compared these data with the unproblematic/healthy parts. In addition to this, we analyzed radiological nonunion level, integrity in the fracture line, reduction continuity, heterotopic bone formation and the development of callus tissue. Nonunion was seen in all patients except 4 patients. Since there was a delay in nonunion, locking screws that aimed at dynamization were removed in these 4 patients. They were the Winquist and Hansen type-4 cases which were statistically locked. In the follow-ups it was seen that in one of the patients who underwent dynamization there was $5 \mathrm{~cm}$ shortening and malunion (valgus deformity). Osteotomy was performed in this patient and by ilizarov external fixator both the angle and shortening was fixed. In the follow-ups, no complications were encountered other than 40 degrees of flexion restriction in the knee.

It was planned to make autogenous spongioz bone graft for three patients whose nonunion did not occur. 2 of the patients accepted grafting; however 1 patient rejected the intervention. The

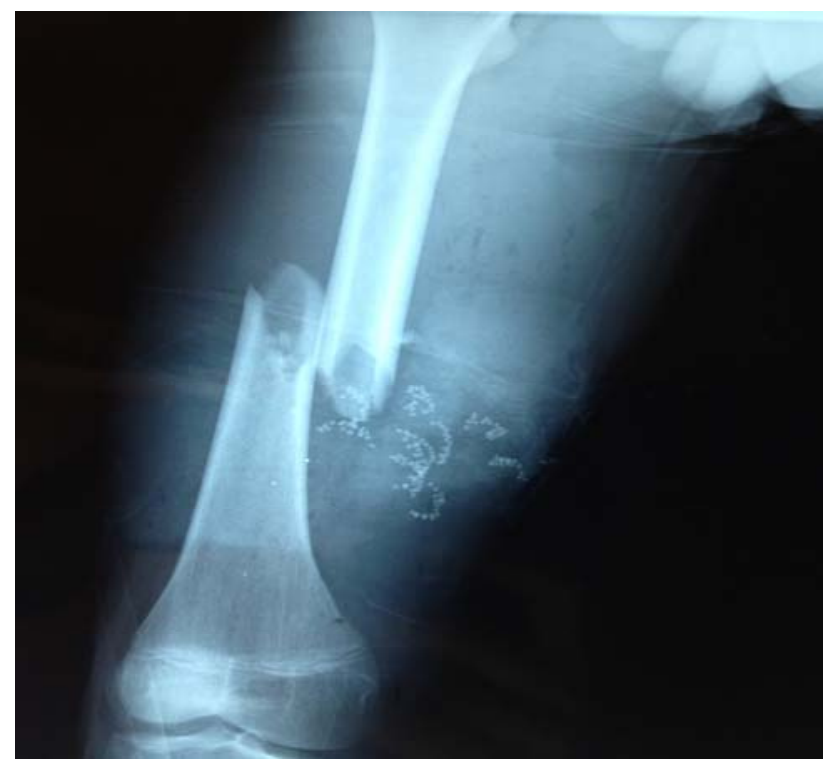

Fig. 1. Preop AP radiography of patients who operated with intramedullary nail. replacement of autogenous spongioz bone graft was materialized in 2 patients and in the followups and it was seen that there was nonunion in these 2 patients. In the 54 year old patient who rejected intervention, nonunion did not develop in the $36^{\text {th }}$ postoperative month. This patient could be mobilized by single crutch. The non-existence of clinical complaints, disappearance of fracture line, painless weight bearing, the presence of callus tissue which would enable the patient full weight bearing in bilateral radiographies were interpreted as positive developments regarding nonunion. The average nonunion time was determined as 16 weeks [10-25] in the Winguist Hansen type 0-1-2 cases and 20.4 weeks [13-32] in the Winguist type 4 cases. When we evaluated the clinical results according to the Thoresen criteria, except one patient, the results of all our patients were assessed as good and excellent (Figure 1.2.3.4).

We believe that after materializing debridement, and wound healing, intramedullary fixation should be applied in type- 2 and type- 3 fractures or external fixation should be applied in these types of fractures.

\section{Discussion}

The big and strong muscles that encircle the femur cause the occurrence of angular and rotational forces on the fragments. This is why providing and maintenance of closed reduction is hard in adults $(2,5,6,7)$. Femur shaft fractures in adults, except some special cases, should be

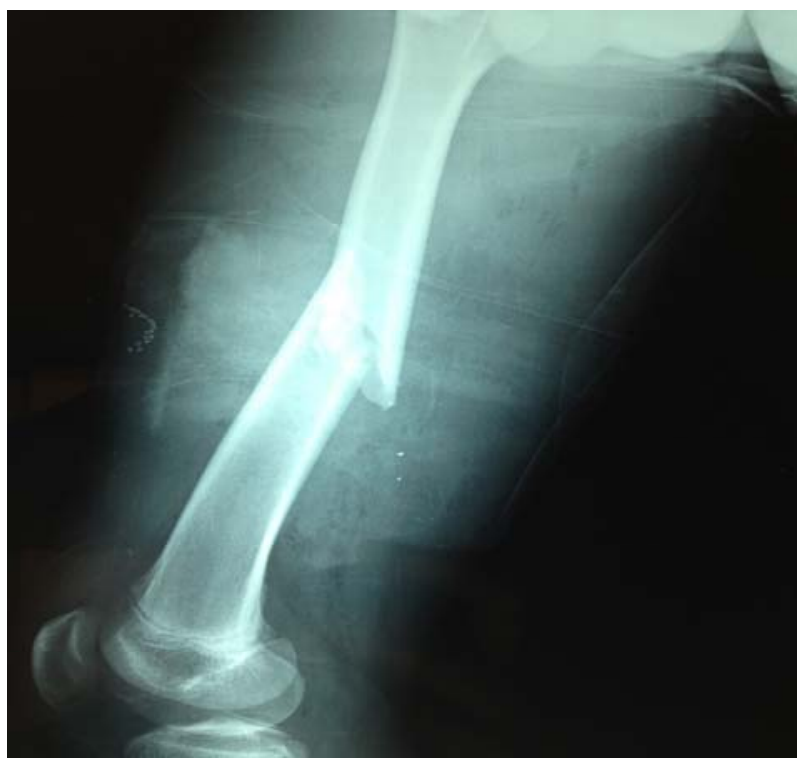

Fig. 2. Preop lateral radiography of patients who operated with intramedullary nail 


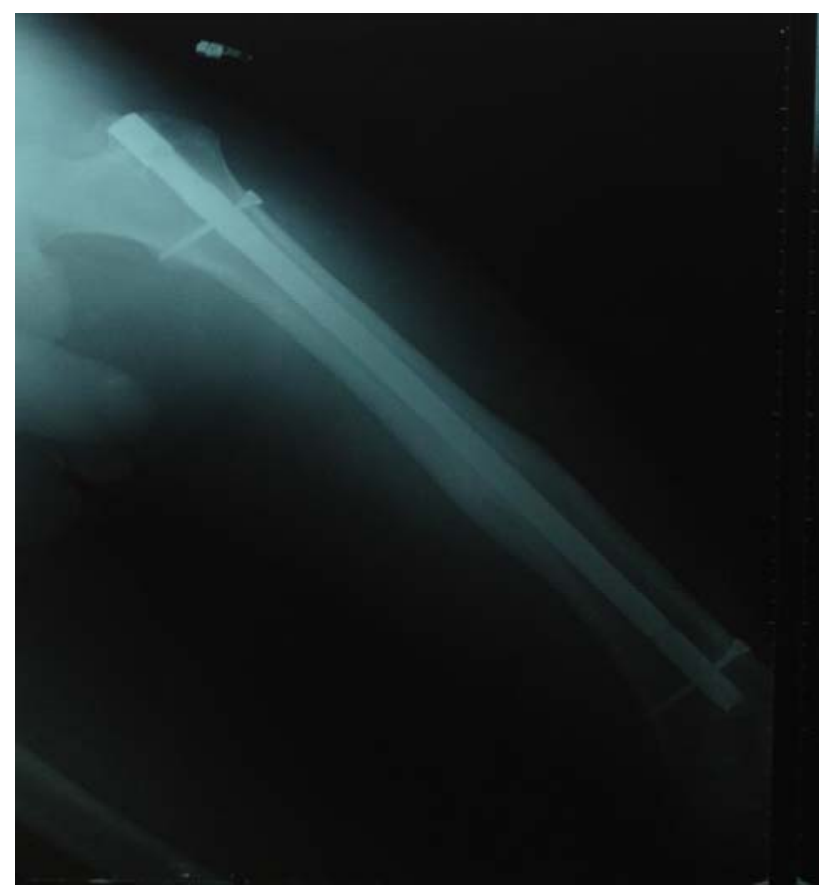

Fig. 3. Control radiography of patients who operated with intramedullary nail.

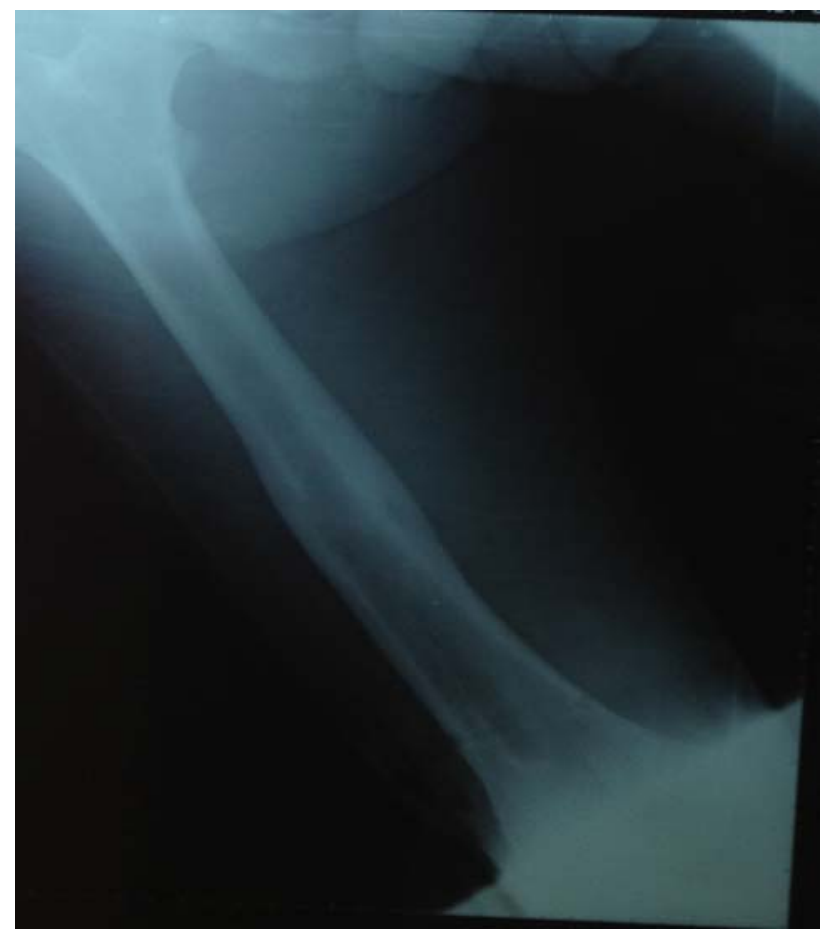

Fig. 4. Control radiography of patients after nail removed.

surgically treated since protracted fixation and immobility can cause severe complications $(2,5,6,9)$. In femur fractures, especially in the middle $1 / 3$ fractures, intramedullary nails are preferred. Various authors have reported that fracture healing rates varied from $82 \%$ to $100 \%$ by the application of intramedullary nailing (6). In our case, in 1 patient, shortening and varus was seen. In this patient, with ilizarov external fixator lengthening was materialized and deformity was fixed. In 2 patients, nonunion occurred after autogenous spongioz grafting. In 1 patient in the $36^{\text {th }}$ postoperative week nonunion did not occur and this patient did not accept additional intervention. When these results are compared with the data in the literature it can be seen that our nonunion rate is comply with the literature.

In lower extremity traumas, femur shaft fractures which are the most common reason for the morbidity and mortality emerge in relation to high-energy traumas to a large extend $(3,10,11)$. These fractures are seen frequently in young adults and this is explained by having an active life-style and so encountering trauma more often at this age range (12).

Bracing is an external fixation device which is used as an option in the treatment of femur shaft fractures. It is used in internal fixation deficiency to neutralize the torsional load which can cause reduction loss. However, the use of the method is restricted even in the best cases that fixation is supplied due to the risks like shortening, angling of varus, overload in the medial compartment of the knee, cosmetic deformity, and motion restriction (12).

On the other hand, it is indicated that external fixators, which are known as another surgical treatment option, provide adequate fixation and stabilization in all shaft fractures including segmental femur fracture (13). When a comparison made between the locked intramedullary nails used in closed femur shaft fractures and external fixation, it has been seen that intramedullary fixation was significantly superior. External fixator may cause infection at the bottom of the screw and knee joint stiffness. In addition, it was understood that it was very hard for the patient to live with external fixation. At the present time, acceptable indications for external fixation are type-3 open fractures, patients with multiple injuries who cannot tolerate anesthesia with long durations and cases which include both fracture and vascular injury. In the literature, it has been reported that in femur shaft fractures, intramedullary nailing can be preferred instead of external fixator considering the superiority of the method in terms of healing and clinical results. Our clinical experience too shows parallelism with the information in the literature on this topic (3).

Plating is another surgical fixation method and it is possible to provide anatomical axe and length in 
femur by this method. However, in $20-30 \%$ of the patients scar in the quadriceps muscle is seen and in relation to this problem, restriction in the motion of knee emerges. In addition to this, a second operation is needed in $5-10 \%$ of the patients due to the insufficiency of fixation. The development of fracture after the application of plate has been reported at a rate of $3 \%$ by Magerl et al. (14) and at a rate of $9 \%$ by Ruedi and Luscher (15). In the locked nailing fracture, although recurrence is quite low, it can observe frequently in the early period after the removal of the nail.

In the treatment with plate, the rate of infection is higher than conservative treatment and intramedullary fixation (16). In the application of plate treatment, devitalization of the cortex, at least in one part, is inevitable. Intramedullary nails have a better control on the femur length and rotation without causing the risk of tissue devitalization. In addition, in intramedullary technique, quadriceps scar, hemorrhage and infection are seen less often in comparison to plating. In clinical and biomechanical studies, there is no need for additional fixation materials due to the stabilization provided by the intramedullary system. However, additional fixation materials are needed especially in the early postoperative period for plate-nail systems (17). Perhaps the only superiority of open reduction and plating to intramedullary nailing is not requiring extensive operating room equipment and scopy.

The smooth and tubular structure of femur shaft is very suitable for intramedullary fixation. When intramedullary nails are compared with plate and external fixators, it is seen that intramedullary nails are closer to body's center of motion and carry lower loads. This mechanical characteristic has various advantages like fewer load on the intramedullary nail, less insufficiency and fatigue, and stimulating remodeling with increased loading (18).

Intramedullary nails have lots of theoretical and practical superiorities in comparison to other external and internal fixation materials. Large surgical exposure and dissection is needed in the application of plate. The fracture hematoma cannot be drained in intramedullary closed nailing and humoral and cellular factors which are important for the wound healing are lost. Thus closed nailing materializes a biological fixation in femur. It has advantages like less surgical dissection, lower infection rate and less quadriceps muscle scar (19).
One of the main advantages of locked intramedullary nailing is high rates of nonunion without complications. These rates are reported between $97-100 \%$ in the literature (18). In our study, the nonunion occurred at a rate of $97.5 \%$. As long as disruption of bone increases (the Winquist Hansen Type-4), nonunion delays.

After intramedullary nailing, fracture healing is swift because in this method nail enables compressive loads to pass fracture zone and so positively affects remodeling and formation of callus in fracture (4). Periosteal circulation which is a main in factor in fracture healing is not damaged or is slightly damaged. There is not an ideal instrument which does not disrupt both endosteal and periosteal circulation and also provide a good mechanical stabilization at the present day. Intramedullary nailing causes the disruption of endosteal circulation; however, periosteal circulation is protected. Swift healing and remodeling begin with the materialization of collateral circulation. The superiority of intramedullary nailing in comparison to conservative and other surgical methods is significant. Poor nonunion is rarely seen. The functional regaining of the abilities is earlier, the length of hospital stay is short and healing process is swift $(13,20,21)$. This rapid rehabilitation process brings along economic advantages since it reduces the length of hospital stay and loss of total workforce.

A recent time ago, in open fractures, intramedullary nailing was not used. Winguist et al. (8) and Brumback et al. (22) have reported that they got successful results by the application of nail after early wound debridement in grade 1-2 open fractures. The conducted clinical studies have revealed that early nailing minimized the risk of infection in open fractures (22). If intramedullary nailing will be used in open fractures, debridement and nailing should be applied as two separate procedures (11).

The infection rates are at a low level after femoral nailing. This rate is higher in open fractures. In different studies, in closed intramedullary nailing, the infection rates have reported less than $2 \%$, and at a rate of $5-10 \%$ in open intramedullary nailing $(22,21)$. In our study, in 1 patient who underwent closed intramedullary nailing, superficial wound site infection developed in the insertion site of the nail. The infection brought under control by superficial wound debridement and antibiotherapy. In none of the patients except this patient, superficial or deep infection developed. 
Rothwell has reported significant shortenings in all femur fractures regardless of the treatment methods (21). However, locked intramedullary nails have decreased this complication rate. Winquist et al. (8) have reported that shortenings $2 \mathrm{~cm}$ and above are seen at a rate of $2 \%$. Also they have stated that in type-1, stabile, short, oblique or transvers fractures, shortening was rarely seen and shortenings below $2 \mathrm{~cm}$ uncommonly caused leg and lumbar pain. According to this, for young patients $1.5 \mathrm{~cm}$, and for patients age 65 and older $2.5 \mathrm{~cm}$ can be considered shortening.

In femur, the same amount of successful results has reported related to reamerized and nonreamerized nailing. In reamerized nailing, less mechanical problems are seen. Pape et al have indicated that in patients both with multiple wounds and lung trauma ARDS rate increased by reamerized (17). Intramedullary nailing should not be preferred in patients with wounding due to the complication of fat embolism (17). According to the data in the literature, reamerized intramedullary nailing operation decreases torsional resistance. Also it has been reported that reamerized intramedullary nailing the diameter of the isthmus bone at a rate of $48 \%$ reduces resistance at a rate of $65 \%$. Besides, it is known that reamerized intramedullary nailing operation increases the operation time and blood loss and causes heat necrosis (9). In all of our cases, reamerized intramedullary nailing operation was materialized in order to increase the mechanical stabilization. However we also avoided excessive reamerized intramedullary nailing operation since it weakens the bone, increases blood loss and causes heat necrosis.

Nerve injury related intramedullary nailing are described for sciatic nerve, peroneal nerve and pudendal nerve. Pudendal nerve palsy is a known complication of intramedullary nailing. This situation also occurs as labile sense changes in females and as scrotal and penile sense changes and sometimes as erectile dysfunctions in males. Pudendal nerve palsy can be avoided by decreasing the pressure at the traction (23). In none of the operations in our cases, traction table was used and neurological complication did not developed in any of our patients.

The development of heterotopic ossification in the soft tissue around proximal femur after femur nailing is known for years (3). Heterotopic bone development associated with the proximal part of the nail is commonly seen. The frequency of heterotopic ossification which was seen in hip after intramedullary nailing had been researched by Brumback et al. (24) The authors have reported that irrigation of bone debris in the soft tissue after nailing did not statistically decrease this frequency. According to the findings, heterotopic ossification was directly related to age, sex, severity of wound, presence of intracranial hemorragia, the level of fracture, the level of disruption, time of nailing, and intramedullary fixation type. Heterotopic ossification can also emerge as a small problem sometimes in gluteal region. It rarely requires surgical intervention. In 12 of our patients, heterotopic ossification was determined in proximal region. However, in any of these patients, there were no complains that emerged due to heterotopic ossification or there were any complains that required intervention.

The opinions related to dynamization have changed a lot in recent years. Brumback et al. (18) have reported that dynamization was not needed and even if the nails were not removed, most of the fractures healed. Browner has showed that in the cases followed up without dynamization, there was not insufficiency or delay during nonunion (3). It has been stated that early dynamization caused shortness, nail protrusion and loss of reduction. Dynamization was applied to 3 patients since nonunion was delayed. In 1 of them, $5 \mathrm{~cm}$ shortness and malunion (15 degrees valgus) occurred. In the other patients nonunion did not occur. Thus cancellous autogenous bone graft was applied and nonunion occurred in the follow-up.

After the treatment of femur shaft fractures by intramedullary nailing, aseptic necrosis at the femur head has been seen (25). Also iatrogenic neck fracture has been reported during nailing. We did not encounter such complications in the femur shaft fractures that we included in this study (16).

We aimed to evaluate early results of locked intramedullary nailing. In light of these evaluations we saw that our results were compatible with the literature. We believe that locked intramedullary nailing is one of the most successful methods in the treatment of femur shaft fractures.

\section{References}

1. Duman E, Ateş Y: The journal of Turkish Orthopaedics and Traumatology Association. 2008; 7: $1-2$.

2. Rockwood and Green's Fraclures in Adults. J. Bi. Lippincott Company, Philadelphia, Third Ed. Vol. 2, 1991.

East J Med Volume:21, Number:1, January-March/2016 
3. Browner BD. Pitfalls, errors, and complications in the use of locking Küntscher nails. Clin Orthop Relat Res 1986; 192-208.

4. Brownwer BD. Locked nailing of femoral fractures. In: Brownwe BD, editör.The science and practice of intramedullary nailing. 2 th ed. Connecticut: Williams \& Wilkins 1996; p. 161-183.

5. Baytok G, Zöhre S. Examination of adult femoral shaft fractures in patients who were treated with conservative methods. Eighth the Turkish orthopedics and traumatology congress book. 14519, Ankara. Printıng press of Emel 1984.

6. Suchalz WR, Jones A. Current concepts review fractures of the shaft of the femur. J bone joint surg 73-:1561-1565-1991.

7. Buxton R.A. The use of perkins' Traction in the treatment of femoral shaft fractures. J Bone joint surg. 63-B:362-366,1981

8. Winquist RA, Hansen ST Jr, Clawson DK. Closed intramedullary nailing of femoral fractures. A report of five hundred and twenty cases.J Bone Joint Surg (Am) 1984; 66: 529-539.

9. Klemm K, Schellmann WD. Dynamic and static locking of the intramedullary nail. Monatsschr Unfallheilkd Versicher Versorg Verkehrsmed 1972; 75: 568-575.

10. Bucholz RW, Jones A. Fractures of the shaft of the femur. J Bone Joint Surg 1991; 73: 1561-1566.

11. Chevalley F, Amsutz C, Bally A. Experimental study of external fixation of femoral fractures. Mechanical properties of different kinds of fixation. Clinical implications. Rev Chir Orthop Reparatrice Appar Mot 1992; 78: 423-429.

12. Kesler SB, Hallfeldt KK, Perren SM, Schweiberer L.The effects of reaming and intramedullary nailing on fracture healing. Clin Orthop 1986; (212): 18-25.

13. Ege R. Femural shaft fractures. Ege R, Traumatology, fractures, joint injuries. Vol. 3, 4 th ed. Ankara: Prıntıng press of Kadığlu 1989. S. 2360-2437.

14. Magerl F, Wyss A, Brunner C, Binder W. Plate osteosynthesis of femoral shaft fractures in adults. A follw-up study. Clin Orthop 1979; (138):62-73.
15. Ruedi TP, Luscher JN. Results after internal fiksation of comminuted fractures of the femoral shaft with DC plates. Clin Orthop 1979;(138): 74-6

16. McKee MD, Waddell JP. İntramedullary nailing of femoral fractures in morbidly obese patients. J Trauma 1994; 36: 208-210

17. Bucholz RW, Brumback RJ. Fractures of the shaft of the femur. In: Rocjwood CA, Green DP, editors. Rockwood and Green's fractures in adults. Vol. 2. 4th ed. Philadelphia: Lippincott; 1996. p. 1827-919.

18. Brumback RJ, Uwagie-Ero S, Lakatos RP, et al. Intramedullary nailing of femoral shaft fractures. Part II: Fracture-healing with static interlocking fixation. J Bone Joint Surg 1988; 70: 1453-1462.

19. Tüzüner $\mathrm{T}$ et al. Treatment of femoral shaft fractures with intramedullary nailing; Acta Orthop Ttraumatol Turc 2002; 36: 211-219.

20. Johnson KD, Johnston DW, Parker B. Comminuted femoral shaft fractures: treatment by roller traction, cerclage wires and an intramedullary nail, or an interlocking intramedullary nail. J Bone joint Surg (Am) 1984; 66: 1222-1235.

21. Rothwell AG. ClosedKuntscher nailing for comminuted femoral shaft fractures. J Bone Joint Surg (Br) 1982; 64: 12-16.

22. Brumback RJ, Ellison PS Jr, Poka A, et al. Intramedullary nailing of open fractures of the femoral shaft. J Bone Joint Surg (Am) 1989; 71: 1324-1331.

23. Stambough JH, Hopson CN, Cheeks ML. Stable and unstable fractures of femoral shaft. Orthop Rev 1991; 20: 855-861.

24. Brumback RJ, Wells JD, Lakatos R, et al. Heterotopic ossification about the hip after intramedullary nailing for fractures of the femur. J Bone Joint Surg 1990; 72: 1067-1073.

25. Mileski RA, Garvin KL, Crosby LA. Avascular necrosis of the femoral head in an adolescent following intramedullary nailing of the femur. A case report. J Bone Joint Surg 1994; 76: 1706-1708. 